\title{
Análise da biometria das espécies florestais teca e mogno submetidas a diferentes tratamentos de irrigação e nutrição mineral
}

\author{
André Luís Teixeira Fernandes ${ }^{1}$ \\ Marianne Fidalgo de Faria ${ }^{2}$ \\ Thaíla de Mello Florêncio² \\ ${ }^{1}$ Engenheiro Agrônomo, Prof. Dr. Universidade de Uberaba e Faculdades Associadas de \\ Uberaba, Av. Nenê Sabino, 1801, 38055-500, Uberaba-MG, andré.fernades@uniube.br \\ ${ }^{2}$ Acadêmicas de Engenharia Ambiental, Universidade de Uberaba, \\ mariannefidalgo@edu.uniube.br e thailamello@gmail.com
}

\section{Study of the impacts and analysis of productivity and development, by irrigation, of legal wood teka and mahogany}

\begin{abstract}
This work presents studies done in the city of Uberaba, MG with the species mahogamy and teka, species of great commercial value, however of medium/long term of growth. The planted forests were appraised with 4,8 years old, where they were exposed to two manners of furnishing water (irrigation and non-irrigation), by a dripping system, and three types of application of fertilizer $(100 \%, 50 \%$ and $25 \%)$. The aim of the project is to analyse the relation costs-benefits of the use of water, as well as the nutrition applicated on it, in the development of forest species, aiming to obtain a more sustainable production of noble wood. The annual medium increment of the studied species was remarkably bigger in the irrigated species. The treatment which has been presenting the better results considering productivity was the one irrigated with $100 \%$ of the necessary application of fertilizer to Mahogany, with 7,5 $\mathrm{m}^{3}$ há $^{-1}$ year $^{-1}$ of growth. As for teca, the best annual increment of wood were obtained with the irrigated parcels, with no difference between the percentages of $50 \%$ and $100 \%$ of necessary nutrition.
\end{abstract}

Palavras-chave: commercial value, forest species, water, development, valor comercial, espécies florestais, água, desenvolvimento.

\section{Introdução}

A região de cerrado possui fatores como baixa fertilidade dos solos, e considerável déficit hídrico que podem ser tidos como limitantes para o desenvolvimento de espécies florestais. Porém, o estado de Minas Gerais, por possuir terras de baixo valor agregado, é a área do Brasil com maior concentração de reflorestamento com espécies de rápido crescimento (MACEDO et al., 2005).

Para obter-se um bom rendimento com o plantio de espécies florestais na região de cerrados deve-se escolher espécies que possuam grande potencial de adaptação e produção na região e atentar-se para a escassez de recursos do meio, que pode gerar intensa competição intra específica (MACEDO et al., 2005).

A Organização das Nações Unidas para a Agricultura e Alimentação (FAO) em sua publicação "Estatísticas Florestais de Hoje para Amanhã" ("Forestry Statistics Today for Tomorrow") prevê que o consumo de madeira roliça para fins industriais vai crescer dos 1,6 bilhões de metros cúbicos $\left(\mathrm{m}^{3}\right)$ registrados em 1991 para 2,6 bilhões de $\mathrm{m}^{3}$ por volta do ano 2010, enquanto que o consumo de madeira serrada vai passar de 456 para 745 milhões de $\mathrm{m}^{3}$, e o de painéis à base de madeira crescerá de 121 para 313 milhões de $\mathrm{m}^{3}$ no mesmo período.

Porém, pressões ambientais se intensificam a cada dia sob a comercialização de madeiras florestais tropicais. Sendo assim, alternativas que visem abastecer o mercado, frente à crescente demanda de madeira, surgem através de resultados de pesquisa e compromisso do setor público e empresarial (FERNANDES; PEREIRA, 1998), e um fato incontestável é que, globalmente, não está havendo replantio das madeiras tropicais no mesmo ritmo em que estão sendo cortadas. 
Os investimentos em reflorestamento não estão acompanhando as taxas de consumo de madeiras tropicais (GRESHAM, 1995).

A teca (Tectona grandis), juntamente com os gêneros Eucalyptus e Pinus, domina cerca de $90 \%$ das plantações florestais nos trópicos. No sudeste asiático, a teca leva de 60 a 80 anos para atingir dimensões de corte, enquanto no Brasil o seu ciclo de corte é de 25 anos. Apesar de seu alto valor agregado (de U\$2000,00/ $\mathrm{m}^{3}$ a $\mathrm{U} \$ 6000,00 / \mathrm{m}^{3}$ ) a teca é uma espécie pouco estudada. Por ser considerada nutricionalmente exigente (IPEF, 2008), obter conhecimentos com relação aos seus aspectos nutricionais é importante para o processo da introdução da teca em qualquer região (BARROSO et al., 2005).

A espécie mogno (Swietenia macrophylla King) possui grande valor de mercado devido às suas propícias características anatômicas e físicas, o que a torna umas das principais fontes de madeira para exportação nos trópicos da América Latina (GUIMARÃES NETO et al., 2004; MELO, CARVALHO, MARTINS, 1989).

O objetivo deste estudo é caracterizar e monitorar o processo de desenvolvimento de duas espécies de grande valor comercial de madeira, teca e mogno, com e sem irrigação, ressaltando a relação custo-benefício, bem como o uso de nutrientes aplicados na água, além de avaliar e criar alternativas quanto ao uso da água no sistema florestal visando propor uma produção mais sustentável das espécies florestais.

\section{Metodologia}

O experimento é conduzido no, cujas coordenadas geográficas são: latitude de 1944'13 'S, longitude $47^{\circ} 57^{\prime} 27^{\prime \prime}$ W e altitude de $850 \mathrm{~m}$.

De acordo com Ranzani (1969), o clima de Uberaba é classificado pelo método de Köppen como Aw, tropical quente úmido, com inverno frio e seco. A precipitação anual é de $1.474 \mathrm{~mm}$ e a temperatura média anual é de $22,6^{\circ} \mathrm{C}$.

O plantio das duas espécies florestais foi realizado em outubro de 2003, no espaçamento de 4,0 x 1,5 m (1667 plantas por hectare), conforme Figuras 1 e 2. O plantio das mudas foi realizado no sulco do subsolador, recomendação do Engenheiro Florestal da empresa Vale do Rio Grande Reflorestamento.

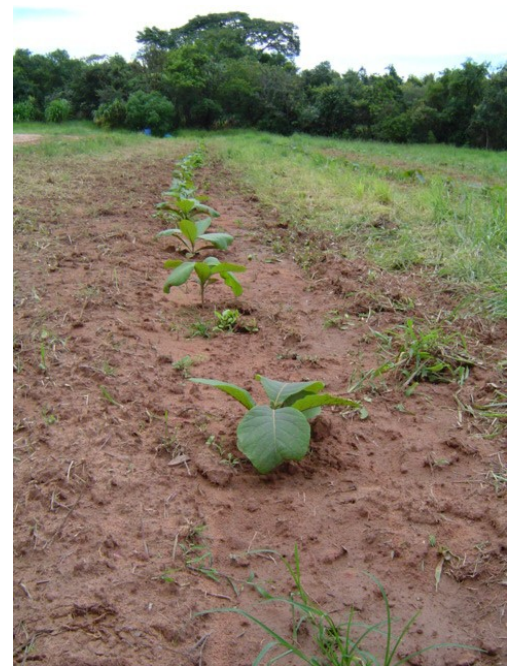

Figura 1: plantio de teca.

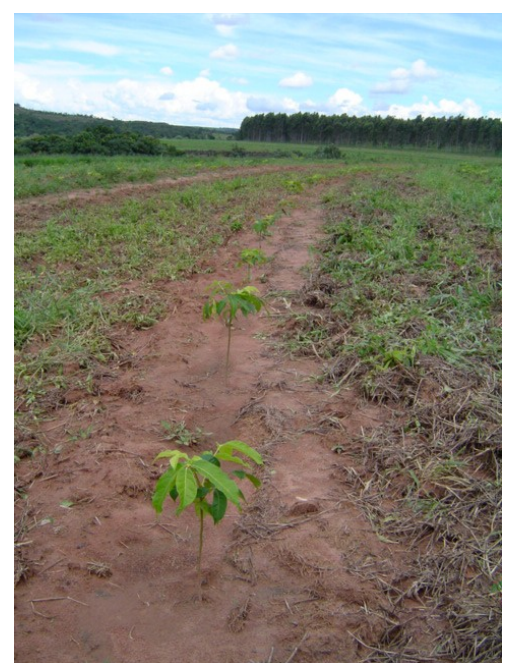

Figura 2: plantio de mogno.

Para a correção do solo, foram utilizadas 2 ton há ${ }^{-1}$ de calcário dolomítico, em área total. Para a adubação de plantio, foram utilizadas as seguintes fontes: sulfato de amônio, yoorin e cloreto de potássio (fórmula 08-28-16). Por planta, foram aplicados $250 \mathrm{~g}$ desta fórmula. A adubação foi dividida em quatro nos diferentes tratamentos sendo que a primeira adubação 
ocorreu aos seis meses, a segunda aos 12 meses, a terceira aos 18 meses e a última aos 24 meses após o plantio das mudas.

O experimento contém dois tipos de fornecimento de água (irrigado e não irrigado), três níveis de adubação (adubação $100 \%$, adubação $50 \%$ e adubação $25 \%$ ) e quatro repetições. A adubação é realizada nos meses de chuva nas parcelas não irrigadas e durante o ano todo nas parcelas irrigadas, por fertirrigação.

O sistema de irrigação utilizado é o por gotejamento, com emissores de 1,6 L h, espaçados a cada $0,75 \mathrm{~m}$. O controle da irrigação é realizado com dados obtidos de estação agrometeorológica automática, por meio dos quais é estimada a evapotranspiração pelo método de Penman Monteith. Para monitoramento da umidade do solo, estão sendo monitoradas baterias de tensiômetros, em três profundidades, 20, 40 e $60 \mathrm{~cm}$.

A área média de cada amostra é de $124,60 \mathrm{~m}^{2}$. Os dados para se realizar os cálculos volumétricos da madeira foram coletados em forma de ziguezague. Para o cálculo volumétrico, foi feita a cubagem rigorosa de 28 árvores das duas espécies, sendo sempre a metade na área irrigada ( 7 árvores) e a outra metade na área não irrigada, totalizando 14 árvores por espécie. Para cada espécie foram cubadas apenas árvores médias (DAP médio, mais ou menos desvio padrão). Para a análise dos dados, foi feita a análise de variância e o teste de comparação de médias. Utilizou-se o hipsometro Eletrônico da Haglof para mensuração das alturas. Utilizouse, também, trena para a mensuração da área das parcelas, coletores de dados com software próprio para as anotações dos dados,além de fita diamétrica, Suta graduada em mm, Motosserra e aparelho GPS.

De todas as árvores cubadas foram retiradas informações através de seções. A altura das seções foi de $0,1 \mathrm{~m}, 0,7 \mathrm{~m}, 1,3 \mathrm{~m}, 2,0 \mathrm{~m}$ e a partir daí de metro em metro, sempre coletando dois diâmetros ortogonais, com suta graduada em mm. Foi coletada também a altura total de cada árvore.

Realizou-se a desrama baixa das amostras de teca e mogno do experimento em todo o talhão, pois nos primeiros anos de plantio, sendo as árvores ainda muito jovens, não há como selecionar os melhores fenótipos nos plantios via mudas. Logo, foi feita a desrama em todas as árvores e esta vem sendo mantida a fim de melhorar a qualidade da madeira, livrando-a de nós e diminuindo a conicidade do tronco.

\section{Resultados}

Das árvores de teca retiradas para cubagem, aos 58 meses de idade, obteve-se um diâmetro mínimo de 9,0 cm e máximo de $11,0 \mathrm{~cm}$ nas amostras irrigadas, e diâmetro mínimo de 7,9 cm e máximo de 10,9 cm nas amostras não irrigadas. Já com o mogno obteve-se um diâmetro mínimo de 8,3 cm e máximo de $10,1 \mathrm{~cm}$ nas amostras irrigadas, e diâmetro mínimo de $2,9 \mathrm{~cm}$ e máximo de $6,9 \mathrm{~cm}$ nas não irrigadas. Estes dados mostram que, nesta primeira análise, as amostras irrigadas apresentaram maior desenvolvimento de caule que as não irrigadas.

Analisando-se o incremento médio anual (I.M.A.) dos tratamentos, em ambas as espécies, as amostras irrigadas apresentaram maiores índices. Quanto à adubação, a $25 \%$ foi a que apresentou melhores resultados, com a teca obtendo uma produção de $14,4 \mathrm{~m}^{3} / \mathrm{ha} /$ ano e o mogno uma produção de $7,5 \mathrm{~m}^{3} / \mathrm{ha} /$ ano, conforme apresentado nas figuras 3 e 4 . . A teca está representada pela letra $\mathrm{T}$, o mogno pela letra $\mathrm{M}$ e as amostras irrigadas aparecem como IR e não irrigadas como NI. Já quanto aos tipos de tratamento, representamos a adubação $100 \%$ pela letra $\mathrm{A}$, a $50 \%$ pela letra $\mathrm{B}$ e a $25 \%$ pela letra $\mathrm{C}$. 


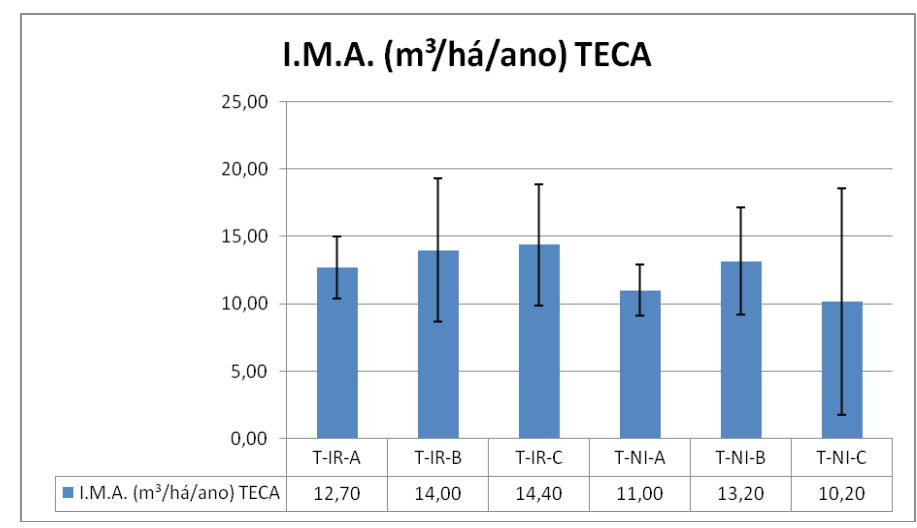

Figura 3: Gráfico de Incremento médio anual (IMA) dos diferentes tratamentos da espécie Tectona Grandis (Teca).

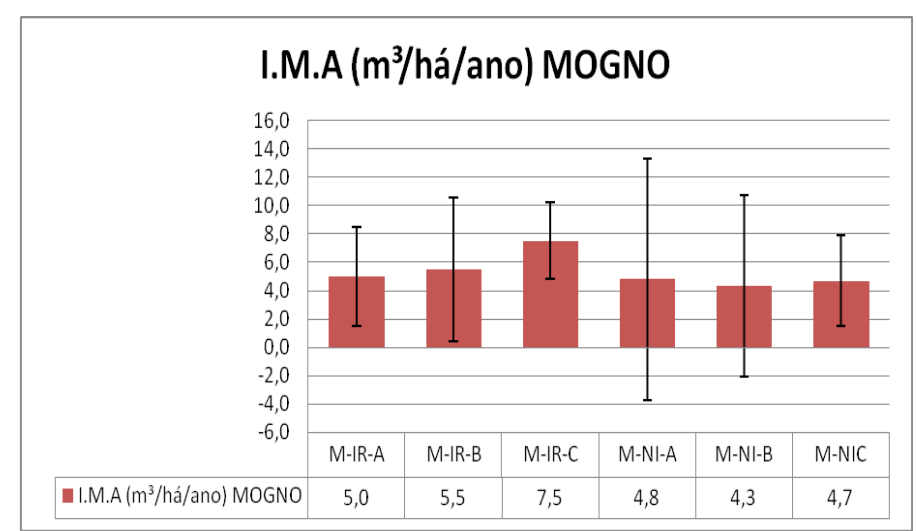

Figura 4: Gráfico de Incremento médio anual (IMA) dos diferentes tratamentos da espécie Swietenia macrophylla (Mogno).

Em relação à altura das árvores analisadas, o tratamento que mostrou melhor resultados, assim como na produção anual (IMA), foi o irrigado e com adubação $25 \%$ que atingiu uma altura média de 10,2 metros para a teca e 5,9 metros para o mogno. Já o tratamento que obteve menos desenvolvimento foi o não irrigado com adubação $25 \%$, também em ambas as espécies. Uma possível explicação para a superioridade dos tratamentos com $25 \%$ da nutrição é que a recomendação foi baseada para suprir as necessidades de eucalipto, que se constitui em espécie com crescimento maior e mais rápido, exigindo maiores quantidades de nutrientes.

Nas Tabelas 1 e 2, constam os resultados estatísticos obtidos com a coleta de dados das amostras, realizada em agosto de 2008 , quando as plantas tinham 4,8 anos de idade.

Tabela 1: Dados estatísticos do mogno: (diâmetro, altura, volume e I.M.A.), obtidos aos 58 meses de idade, Fazenda Escola da Uniube, Uberaba-MG

\begin{tabular}{|c|c|c|c|c|c|c|}
\hline \multirow[t]{2}{*}{ Tratam. } & \multirow[t]{2}{*}{ Estatíst. } & \multirow{2}{*}{$\begin{array}{l}\text { DG } \\
(\mathrm{cm})\end{array}$} & \multicolumn{2}{|c|}{ Alturas (m) } & \multirow{2}{*}{$\begin{array}{l}\text { Volume } \\
\mathrm{m}^{3} / \mathrm{ha}^{-1}\end{array}$} & \multirow{2}{*}{$\begin{array}{c}\text { I.M.A. } \\
\mathrm{m}^{3} \mathrm{ha}^{-1} \mathrm{ano}^{-1}\end{array}$} \\
\hline & & & Méd & Dom. & & \\
\hline \multirow[t]{3}{*}{ M-IR-A } & Médias: & 8,2 & 5,4 & 6,8 & 24,1 & \multirow[t]{3}{*}{5,0} \\
\hline & C.V.\% & 4,4 & 5,5 & 3,0 & 44,6 & \\
\hline & I.C. $95 \%$ & 6,9 & 8,8 & 4,8 & 71,0 & \\
\hline \multirow[t]{3}{*}{ M-IR-B } & Médias: & 8,3 & 5,3 & 6,7 & 26,9 & \multirow[t]{3}{*}{5,5} \\
\hline & C.V.\% & 20,6 & 19,5 & 8,4 & 57,5 & \\
\hline & I.C. $95 \%$ & 32,8 & 31,1 & 13,3 & 91,5 & \\
\hline \multirow{3}{*}{ M-IR-C } & Médias: & 9,1 & 5,9 & 7,7 & 36,6 & \multirow[t]{3}{*}{7,5} \\
\hline & C.V.\% & 7,2 & 6,9 & 5,1 & 22,1 & \\
\hline & I.C. $95 \%$ & 11,4 & 11,0 & 8,1 & 35,2 & \\
\hline
\end{tabular}




\begin{tabular}{llccccc} 
M-NI-A & Médias: & 8,0 & 5,1 & 6,5 & 23,3 & 4,8 \\
& C.V.\% & 17,6 & 15,6 & 9,3 & 71,7 & \\
& I.C. $95 \%$ & 43,7 & 38,7 & 23,1 & 178,0 & \\
\hline M-NI-B & Médias: & 7,6 & 5,3 & 7,0 & 21,0 & 4,3 \\
& C.V.\% & 8,6 & 3,8 & 8,0 & 59,1 & \\
& I.C. $95 \%$ & 21,4 & 9,4 & 19,8 & 146,8 & \\
\hline \multirow{2}{*}{ M-NI-C } & Médias: & 7,4 & 4,8 & 5,9 & 12,9 & 2,7 \\
& C.V.\% & 11,4 & 12,7 & 10,2 & 48,4 & \\
& I.C. & 28,4 & 31,4 & 25,3 & 120,2 & \\
\hline
\end{tabular}

Legenda: M - mogno; IR - irrigado; NI - não irrigado; A - adubação 100\%; B - adubação 50\%; C adubação $25 \%$

Tabela 6: Dados estatísticos da teca (diâmetro, altura, volume e I.M.A.), obtidos aos 58 meses de idade, Fazenda Escola da Uniube, Uberaba-MG

\begin{tabular}{|c|c|c|c|c|c|c|}
\hline \multirow[t]{2}{*}{ Tratam. } & \multirow[t]{2}{*}{ Estatíst. } & \multirow{2}{*}{$\begin{array}{l}\mathrm{DG} \\
(\mathrm{cm}) \\
\end{array}$} & \multicolumn{2}{|c|}{ Alturas (m) } & \multirow{2}{*}{$\begin{array}{l}\text { Volume } \\
\mathrm{m}^{3} \mathrm{ha}^{-1}\end{array}$} & \multirow{2}{*}{$\begin{array}{c}\text { I.M.A. } \\
\mathrm{m}^{3} \mathrm{ha}^{-1} \mathrm{ano}^{-1}\end{array}$} \\
\hline & & & Méd. & Dom. & & \\
\hline \multirow{3}{*}{ T-IR-A } & Médias: & 9,9 & 9,4 & 11,1 & 61,5 & 12,7 \\
\hline & C.V.\% & 3,8 & 3,2 & 3,0 & 11,5 & \\
\hline & I.C. $95 \%$ & 6,0 & 5,1 & 4,8 & 18,2 & \\
\hline \multirow[t]{3}{*}{ T-IR-B } & Médias: & 10,3 & 9,8 & 11,3 & 67,9 & 14,0 \\
\hline & C.V.\% & 8,4 & 12,4 & 9,9 & 23,8 & \\
\hline & I.C. $95 \%$ & 13,3 & 19,8 & 15,7 & 37,8 & \\
\hline \multirow[t]{3}{*}{$\overline{\text { T-IR-C }}$} & Médias: & 10,6 & 10,2 & 11,4 & 69,7 & 14,4 \\
\hline & C.V.\% & 5,4 & 6,6 & 7,5 & 19,8 & \\
\hline & I.C. $95 \%$ & 8,7 & 10,5 & 12,0 & 31,5 & \\
\hline \multirow[t]{3}{*}{ T-NI-A } & Médias: & 10,3 & 8,9 & 10,6 & 53,3 & 11,0 \\
\hline & C.V.\% & 1,3 & 12,1 & 8,0 & 10,7 & \\
\hline & I.C. $95 \%$ & 2,0 & 19,3 & 12,7 & 17,0 & \\
\hline \multirow[t]{3}{*}{ T-NI-B } & Médias: & 10,4 & 9,5 & 11,1 & 64,0 & 13,2 \\
\hline & C.V.\% & 4,5 & 5,4 & 4,0 & 19,1 & \\
\hline & I.C. $95 \%$ & 7,2 & 8,6 & 6,3 & 30,3 & \\
\hline \multirow[t]{3}{*}{ T-NI-C } & Médias: & 9,4 & 8,2 & 9,8 & 49,5 & 10,2 \\
\hline & C.V.\% & 13,1 & 26,7 & 15,3 & 50,7 & \\
\hline & I.C. $95 \%$ & 20,9 & 42,4 & 24,3 & 80,7 & \\
\hline
\end{tabular}

Legenda: T - teca; IR - irrigado; NI - não irrigado; A - adubação 100\%; B - adubação 50\%; C adubação $25 \%$

A variância entre os tratamentos foi comprovada por testes de normalidade KS e Lilliefors, homocedasticidade pelo Teste de Bartlett e variância pelo Teste de Fisher (Anova One Way) realizados com o CAP, DAP e altura das espécies.

É importante ressaltar que as amostras de mogno tiveram seu desenvolvimento prejudicado no experimento em função do ataque de larvas Hypsypyla grandella, conhecidas como "broca", o que explica a maior resposta da teca à irrigação apesar de ser nutricionalmente mais exigente que o mogno. Apesar do acontecido, as amostras irrigadas também se mostraram com maior grau de desenvolvimento que as não irrigadas.

Dentre as espécies, teca e mogno, do experimento, apenas a Teca apresentou medidas apropriadas para desbaste de acordo com o trabalho de França (1989), que estipula as medidas mínimas do diâmetro basal para desbaste de $15 \mathrm{~cm}$, para madeiras destinadas para serraria, e quatro centímetros, para madeiras destinadas para processamento mecânico. Logo, será feito o 
desbaste de 55\% das árvores de teca. Já quanto ao mogno, será necessário aguardar que o diâmetro basal atinja medidas suficientes para o primeiro desbaste.

Tendo por base o preço da teca de $\mathrm{R} \$ 700,00 / \mathrm{m}^{3}$, de acordo com o diâmetro e volume apresentados pelas amostras com 4,8 anos de idade, obtêm-se, com o volume total de madeira do experimento, uma quantia de $\mathrm{R} \$ 59.439,00$ há $^{-1}$ para o tratamento irrigado com $25 \%$ de irrigação, que apresentou os melhores resultados em produtividade. Enquanto o tratamento não irrigado com $25 \%$ de adubação, que apresentou os piores resultados, renderia, com 4,8 anos de idade, um total de $\mathrm{R} \$ 43.277,00$ há $^{-1}$, resultando em uma diferença de $\mathrm{R} \$ 16.162,00 /$ há $^{-1}$ entre os dois tratamentos.

Para obtermos uma quantia anual obtida com a produção de madeira da teca dividimos o

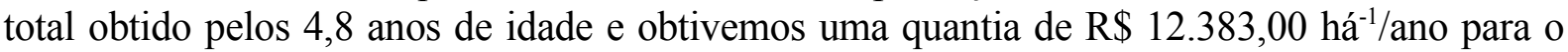
tratamento irrigado com $25 \%$ de irrigação.

É importante ressaltar que não foi feita a avaliação econômica do mogno por este não apresentar medidas de diâmetro basal suficientes para o primeiro desbaste e, sendo assim, não haver necessidade de venda da madeira nesta idade, já que o desbaste somente será feito quando os espécimes apresentarem diâmetro basal suficiente para tal.

\section{Conclusões}

A análise dos dados obtidos nos mostrou que o uso da água é vantajoso em nível de produção e desenvolvimento em espécies florestais de fibra longa e médio/longo crescimento. Tanto no mogno quanto na teca as amostras com adubação $25 \%$ foram as que mostraram mais resposta ao tratamento, apesar de o desenvolvimento do mogno ter sofrido interferência do ataque de larvas Hypsypyla grandella.

A variação de desenvolvimento dentre os tratamentos foi significativa entre os tratamentos irrigados e os não irrigados, sendo que os irrigados foram os que obtiveram os maiores índices de incremento médio anual (IMA). A variância foi comprovada pelos testes de normalidade, homocedasticidade e Fisher feitos posteriormente com o CAP, DAP e altura das espécies.

Comparando-se a diferença financeira ( $\mathrm{R} \$ 16.162,00 /$ há $^{-1}$ ) entre o melhor, irrigado com $25 \%$ de irrigação, e o pior, não irrigado com $25 \%$ de irrigação, tratamento de teca é seguro afirmar que a irrigação desta espécie é viável tanto em termos financeiros quanto em termos de produtividade.

\section{Referências}

BARROSO, Deborah Guerra, et al. Diagnóstico de deficiências de macronutrientes em mudas de teca. Revista Árvore, Viçosa, v. 29, n. 5, p.671-679, ago. 2005.

FAO. Forestry: statistics today for tomorrow. Roma, Itália. 39p.

FERNANDES, V. T.; PEREIRA, R. S. Comportamento Ecofisiológico do Mogno (Swietenia macrophylla, King) no município de Miguel Pereira, RJ. Disponível em: <http://br.geocities.com/floramrural/0130.pdf> . 1998. Acesso em: 10 abr 2008.

FINGER, Z.; FINGER, F. A.; DRESCHER, R. Teca (Tectona Grandis): plante esta idéia. In: Simpósio Brasileiro de Pós Graduação em Engenharia Florestal, 1., 2001, Santa Maria-RS. Anais... Santa Maria: UFSM, 2001.

FRANÇA, F.S.. Efeito do número de árvores remanescentes na produção de madeira de Eucalyptus saligna Smith em segunda rotação. 1989. 138 p. Tese Mestrado, ESALQ/USP, Piracicaba, 1989.

GRESHAM, Gordon E.. Mercado de madeira de florestas plantadas. Anais do Seminário Internacional de Utilização da Madeira de Eucalipto para Serraria. São Paulo: Usp, 1995. p. 147 - 165. v. 1.

GUIMARÃES NETO, Assis Brasil et al. Avaliação do plantio homogêneo de mogno, Swietenia macrophylla King, em comparação com o plantio consorciado com Eucalyptus urophylla S. T. Blake, após 40 meses de idade. Revista Árvore, Viçosa, v. 29, n. 6, p.777-784, ago. 2004. 
INSTITUTO DE PESQUISAS E ESTUDOS FLORESTAIS. Identificação de Espécies Florestais: Tectona grandis (Teca). [Desenvolvido pelo Instituto de Pesquisas e Estudos Florestais - IPEF], 2008. Disponibiliza informações sobre o ambiente florestal. Disponível em: $<$ http://www.ipef.br/identificacao/tectona.grandis.asp $>$. Acesso em: 28 dez. 2008.

MACEDO, Renato Luiz Grisi et al. Desenvolvimento inicial de Tectona Grandis L. f. (Teca) em diferentes espaçamentos no município de Paracatu, MG. Cerne, Lavras, v. 11, n. 1, p.61-69, jan./mar. 2005.

MELO, J. E.; CARVALHO, G. M.; MARTINS, V. A. Espécies de madeiras substitutas do mogno. Manaus: IBAMA, 1989. 16p. (Série técnica, 6).

RANZANI, G. Manual de levantamento de solos. São Paulo: Ed. Edgard Blucher Ltda., 1969. 167p. 